\title{
CAPACIDADES DINÂMICAS E DESEMPENHO MERCADOLÓGICO FRENTE ÀS MÍDIAS SOCIAIS: UM ESTUDO DAS MICRO, PEQUENAS E MÉDIAS EMPRESAS
}

Silvia Spagnol Simi Dos Santos ${ }^{1}$

Sérgio Begnini ${ }^{1}$

Carlos Eduardo Carvalho ${ }^{1}$

${ }^{1}$ Universidade do Oeste de Santa Catarina (UNOESC) 


\section{CAPACIDADES DINÂMICAS E DESEMPENHO MERCADOLÓGICO FRENTE ÀS MÍDIAS SOCIAIS: UM ESTUDO DAS MICRO, PEQUENAS E MÉDIAS EMPRESAS}

Resumo: O objetivo deste estudo é analisar o impacto do uso das mídias sociais nas capacidades dinâmicas e na performance mercadológica das micro, pequenas e médias empresas atuantes no estado de Santa Catarina, Brasil. A metodologia adotada foi quantitativa e descritiva, com coleta de dados por meio de uma survey. A análise dos dados foi realizada sobre uma amostra de 143 empresas, utilizando-se da análise fatorial realizada com o software $\operatorname{SPSS}^{\circledR}$ e da modelagem de equações estruturais por meio do software AMOS $^{\circledR}$. Os resultados das hipóteses testadas apontam para o efeito positivo entre o uso de mídias sociais e capacidades dinâmicas e entre o uso de mídias sociais e o desempenho mercadológico. Porém, as capacidades dinâmicas não se mostraram mediadoras do relacionamento entre o uso das mídias sociais e o desempenho mercadológico. O maior desafio para os gestores destas empresas é transformar os dados das MS em conhecimentos pessoais valiosos e explorar esses insights para criar valor agregado é a maior barreira destas empresas.

Palavras-chave: Capacidades dinâmicas. Desempenho Mercadológico. Mídias Sociais. MPMEs.

\section{Introdução}

O propósito deste estudo é a abordagem das Capacidades Dinâmicas (CD) na gestão estratégica focada em processos relacionados ao uso das Mídias Sociais (MS) no que tange o Desempenho Mercadológico (DM) das empresas. O pressuposto é que em ambientes altamente dinâmicos como o das mídias sociais, as CD consistem de processos de tomada de decisão estratégica que manipula os recursos para a obtenção de valor (EISENHARDT; MARTIN, 2000).

Mídias sociais são "sites na internet construídos para permitir a criação colaborativa de conteúdo, a interação social e o compartilhamento de informações em diversos formatos”, ou seja, uma empresa estar nas mídias sociais é permitir conversações. (TELLES, 2011, p. 19). Neste contexto, o presente estudo foi delimitando às micro, pequenas e médias empresas (MPMEs) pela popularização e ambiente turbulento das MS e de sua utilização nas empresas. Destaca-se as mudanças tecnológicas da área das MS na última década, as quais propiciam dinamicidade ao ambiente corporativo.

As estratégias e táticas que as MS oferecem são inúmeras. Diante disso, a questão central desta investigação é: o uso das MS por MPMEs catarinenses gera uma relação positiva com as CD? As MS geram desempenho mercadológico em empresas? E essas CD geram

Organizadores:

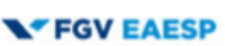

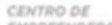

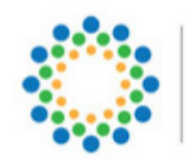

ANEGEPE

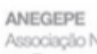

Realizadores:
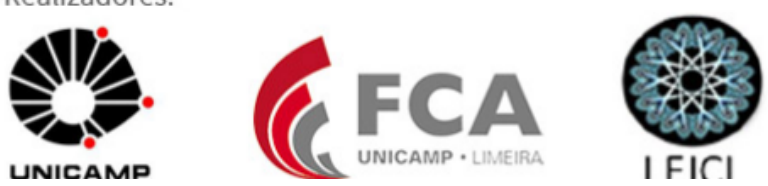
desempenho? O objetivo da pesquisa é mensurar a relação entre CD e DM a partir do uso das MS por MPMEs do estado de Santa Catarina, Brasil.

Como metodologia, optou-se pela quantitativa por intermédio de aplicação de um questionário survey e análise multivariada. Esta investigação faz sentido ao mesmo tempo em que evoca direcionamentos futuros para estudos das próprias empresas pela dinamicidade do mercado. O estudo possibilita ainda avançar teórica e empiricamente na busca de fatores que permitem maximizar a produtividade das organizações.

$\mathrm{O}$ artigo está dividido em cinco seções. Na seção 2 encontra-se uma revisão bibliográfica a respeito das $\mathrm{MS}, \mathrm{CD}, \mathrm{DM}$ e as hipóteses, na seção 3 os procedimentos metodológicos, na seção 4 apresentamos a análise dos resultados e, na seção 5 , as considerações finais.

\section{Referencial Teórico e Hipóteses da Pesquisa}

\subsection{Uso das MS e geração de CD em MPMEs}

$\mathrm{O}$ relacionamento entre MS e CD tem sido abordado sob diversos aspectos pela literatura da área. Para citar algumas pesquisas recentes, por exemplo, os trabalhos de Zavattaro e Daspit (2016) abordam a inovação, de Pogrebnyakov (2017) e Pinho e Prange (2016) abordam a internacionalização das empresas. Outros trabalhos, como o de Braganza et al. (2017) abordou o gerenciamento, de Braojos-Gomes et al. 2015 e Wieneke et al. (2016) focaram no retorno de ativos e ainda Liu et al. (2015) e Erevelles et al. (2016) abordaram marketing e orientação para o mercado.

Em outros estudos recentes, estão relacionadas ainda as capacidades exigidas das empresas para transformar os dados das MS em conhecimentos pessoais valiosos e explorar esses insights para criar valor agregado para os clientes (WIENEKE; LEHRER, 2016); ao uso das MS no contexto do marketing $b 2 b$ a partir de uma perspectiva de capacidade organizacional dinâmica (WANG et al., 2017); as iniciativas de mídia social enquanto impactos positivos sobre a eficiência operacional e a inovação. (LAM et al., 2016).

Nesse ambiente, entende-se que as MS modificaram a forma de como uma organização está relacionada com seus stakeholders, levando a diferentes possibilidades em várias funções organizacionais, do marketing ao gerenciamento de operações e ao desenvolvimento de novos produtos. (LAM et al., 2016). Portanto, a imersão de empresas nas MS pode mudar a configuração de como as organizações se relacionam com seus stakeholders pois as redes sociais fornecem uma plataforma para compartilhamento de experiências, acumulação de conhecimento e aprendizado organizacional (NGUYEN et al., 2015).

Seu uso pode tornar-se uma fonte decisiva de DM pois já estamos vivendo numa cultura da convergência (JENKIS, 2009, P. 31) e essa cultura transforma "a prática das empresas em sua relação com fornecedores, compradores, em sua administração, em seu processo de
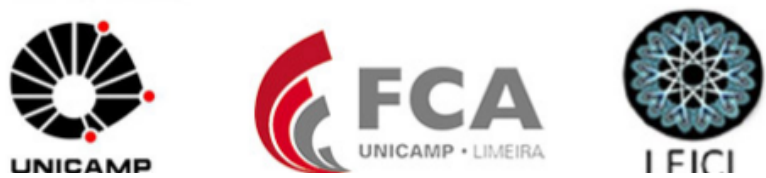
produção e em sua cooperação com outras empresas, em seu financiamento e na avaliação das ações em mercados financeiros" (CASTELLS, 2003, p. 56).

Da mesma forma, seu mal-uso, também acarreta resultados na performance das empresas. Neste contexto, pesquisas contemporâneas já se interessaram em como os empreendedores das MPMEs com capacidades inadequadas e recursos limitados conduzem transformação ao comércio eletrônico transfronteiriço (CBECO) na China, por exemplo (LI et al., 2017). O estudo revelou as etapas-chave no processo, incluindo "renovação de cognição gerencial, desenvolvimento de redes sociais gerenciais, construção de equipe de negócios da CBEC e criação de capacidade CBEC organizacional”. (LI et al., 2017, p. 21, tradução nossa).

Por exemplo, Braojos-Gomez et al. (2015) descobriram que os mecanismos fundamentais através dos quais as pequenas empresas aprendem a desenvolver uma competência em redes sociais são por intermédio da capacidade da infra-estrutura de TI, da pressão do concorrente social, do gerenciamento de marketing e da gestão da inovação. Para Teece et al. $(2014,167)$, o modelo que sugere a criação de riqueza em regimes de "alta mudança tecnológica depende em grande medida da capacidade tecnológica, organizacional e de processos gerenciais da empresa".

Esse conjunto que envolve as MS às MPMEs é volátil, portanto a questão fundamental é como e se essas empresas alcançam e mantêm DM por intermédio das CD absorvidas das MS e quais os caminhos percorridos positiva ou negativamente dessas empresas foram utilizados para instituir a melhor estratégia ou prática empresarial. Evidentemente as CD tem esse objetivo de destacar a exploração das competências internas e externas da empresa para enfrentar esse ambiente de mudança (TEECE et al., 2014). Neste ambiente, surge a seguinte hipótese de pesquisa:

Hipótese 1: O uso de MS influencia positivamente as CD, ou seja, quanto maior o uso de MS maior serão as CD da empresa.

\subsection{Influência de MS e CD no desempenho mercadológico}

Capacidades Dinâmicas são entendidas como as estratégias desenvolvidas pela empresa, por meio das quais, os gestores acessam os recursos, seja adquirindo, criando, e os alocam, gerando novas estratégias, e criando valor para a organização (EISENHARDT; MARTIN, 2014) possibilitando desenvolver diferenciais de mercado, frente à concorrência. Assim, os gestores têm maiores possibilidades para integrar, construir e reconfigurar, tanto as competências internas, quanto as competências externas da organização. As capacidades dinâmicas constituem-se então, em fontes de vantagem competitiva sustentável para as organizações (EISENHARDT; MARTIN, 2014).

As CD possibilitam que a empresa, atuando em ambientes complexos e de rápidas mudanças, possa detectar, analisar e entender as fontes e os procedimentos que envolvem a

\section{Organizadores:}

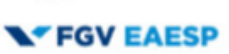

\section{CONTRO of}

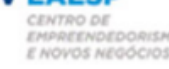

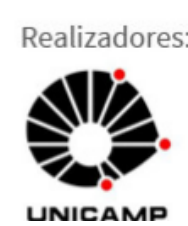


criação e apropriação de riquezas (TEECE et al., 2014). O estudo das CD, auxilia a entender o modo pelo qual as empresas agem para reconfigurar seus recursos, em um ambiente de incertezas e turbulento, ou mesmo em ambientes munificentes, buscando elevado desempenho mercadológico. Assim, a relação das CD com a vantagem competitiva, está fundamentada na possibilidade, da empresa criar, configurar, integrar e combinar seus recursos (ZOLO; WINTER, 2002; WANGE; AHMED, 2007; EISENHARDT; MARTIN, 2014).

As CD sustentam que, a competência das empresas em mudar e reorganizar novos recursos, em diferentes configurações, torna-se mais relevante do que os próprios recursos, para a geração da vantagem competitiva sustentável (TEECE et al., 2014), portanto, as CD alteram também a base de recursos das organizações por intermédio de estratégias organizacionais. Teece, Pisano e Shuen (2014) destacam que o universo da estratégia empresarial fornece uma vantagem competitiva, sustentada pelo desempenho da organização através das várias rotinas organizacionais desempenhadas pelas pessoas, e conforme aumenta a incidência dessas rotinas é possível desenvolver competências organizacionais. A competência organizacional acontece nas empresas quando as pessoas passam a ter consciência do resultado gerado por suas ações no desempenho geral da organização, por meio dos recursos que utilizam (ZOLLO; WINTER, 2002).

É com base no conjunto de recursos disponíveis, e na reconfiguração destes que a empresa poderá alcançar resultados positivos. Aliás, a busca por obter resultados positivos é constante em todas as empresas, pois, é com base nos resultados alcançados, que haverá ou não, sustentabilidade no mercado. Na literatura é possível encontrar vários estudos que buscam compreender a dinamicidade das empresas em relação ao mercado, sendo que vários deles focam no desempenho das organizações. Seja com fundamento no posicionamento estratégico (PORTER, 1980), ou na visão baseada em recursos da empresa (VBR) (BARNEY, 1991; PETERAF, 1993), ou ainda com fulcro nas CD (TEECE; PISANO; SHUEN., 1997; EISENHARDT; MARTIN, 2000), o desempenho das empresas é assunto de pesquisas, debates e aprofundamentos.

Diante da concorrência, do ambiente complexo e das incertezas que se estabelecem no mercado, as empresas necessitam munir-se de elementos, que possibilitem obter vantagem competitiva sustentada, e desta forma, atingir elevado desempenho (MARTYNOW; SHAFTI, 2016). Um dos fatores que impactam diretamente no alcance do desempenho de mercado é a maturidade da organização, fruto da trajetória e de constantes revisões e melhorias dos processos internos (BARRA; LADEIRA, 2017). Com isso, a implantação de estratégias diferenciadas, com base em capacidades e recursos raros, inimitáveis, de valor, e organizados, a empresa (BARNEY; HESTERLY, 2007) poderá gerar valor ao cliente, e obter desempenho superior, no mercado.

Utilizando alguns dados e algumas informações torna-se possível identificar e mensurar o DM das empresas. Tentando realizar tal trabalho, alguns autores vêm, ao longo do

\section{Organizadores:}

NFGV EAESP

corrao or

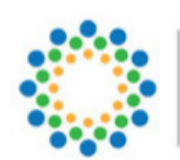

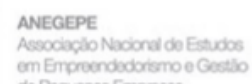
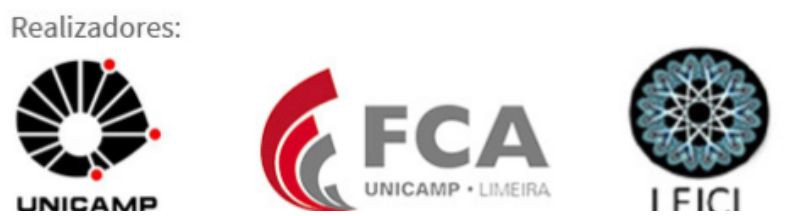
tempo, identificando e também utilizando, diferentes variáveis: a satisfação dos clientes, a satisfação dos funcionários, a lucratividade, o crescimento das vendas e a reputação da empresa (VENKATRAMAN; TAMANUJAM, 1986); capacidade de produção, evolução no número de cliente, fidelização dos clientes, investimento em treinamentos, propaganda e modernização, qualidade do produto/serviço, valor da marca, volume de vendas (CALLADO et al., 2008); volume de vendas e a tomada de decisão (LOMBARDI et al., 2010); fidelização dos clientes, atração de novos clientes, volume de vendas, Market share e satisfação dos clientes (SANTOS et al., 2014).

Com esses indicativos, percebe-se que para atingir um DM favorável, as empresas necessitam desenvolver $\mathrm{CD}$, isto é, aprender e reinventar-se constantemente. Precisam ser capazes de atrair novos clientes, reter os atuais, aumentar a lucratividade, bem como a participação no mercado, além de oferecer maior qualidade, preços atraentes e atendimento personalizado (CHRISTENSEN, 1997; OECD, 2005; TIDD et al., 2005; TIGRE, 2006; CHESBROUGH, 2012). A literatura pesquisada e apresentada identifica que, ao desenvolver $\mathrm{CD}$, as empresas estarão mais preparadas para competir e atuar no mercado, possibilitando obter maior desempenho mercadológico.

Estudos recentes demonstram haver um relacionamento entre MS e desempenho, como Gupta e George (2016), Lam et al. (2016), Wang e Kim (2017), Wamba et al. (2017) e Ahani et al., 2017). Assim, é possível elaborar as seguintes hipóteses de pesquisa:

Hipótese 2: O uso de MS influencia positivamente o desempenho, ou seja, quanto maior o uso de MS maior será o desempenho da empresa.

Hipótese 3: CD influenciam positivamente o desempenho, ou seja, quanto maior as CD maior será o desempenho da empresa.

A Figura 1 propõe o modelo de pesquisa, com os construtos de estudo e seus relacionamentos.

Figura 1 - Estrutura Teórico-Empírica

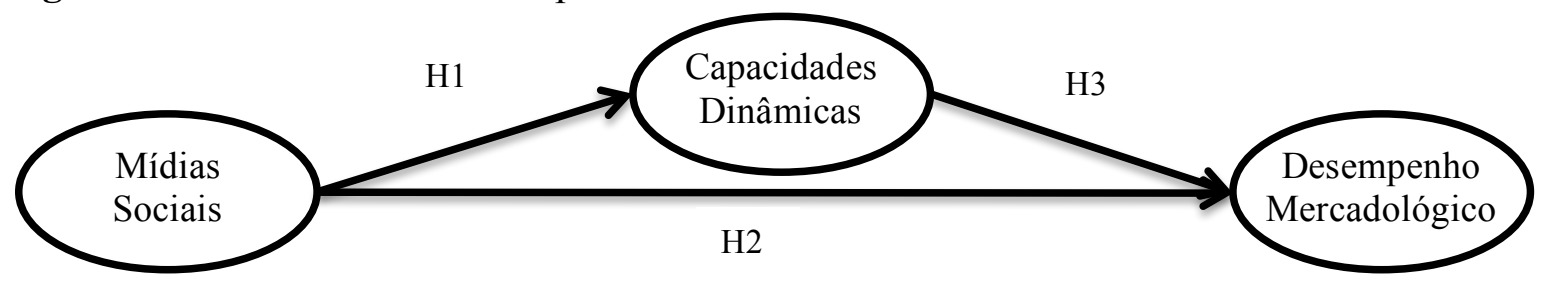

Fonte: os autores (2018)

Organizadores:

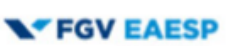
COWTino or

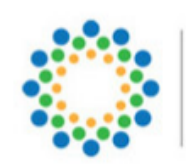
ANEGEPE
Associaglo Naciondi do Estudos m Emproendadorismo e Gestlo de Poqueras Empresas
Realizadores:
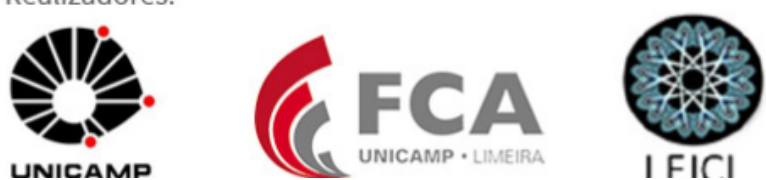


\section{Procedimentos Metodológicos}

\subsection{Amostra e coleta de dados}

Este estudo foi realizado com micro, pequenas e médias empresas, que integram a Federação das Associações Empresariais de Santa Catarina, Brasil. Foram obtidas 172 respostas, das quais 143 passaram a integrar a amostra do estudo, pois estavam completas e atendiam os critérios da pesquisa. A pesquisa configura-se como descritiva e quantitativa, uma vez que busca medir a relação do uso das mídias sociais nas capacidades dinâmicas, e a relação com o desempenho mercadológico.

Tratam-se de dados primários, coletados por uma survey de 26 questões, aplicada por meio do Survey Monkey. Quatro questões foram de identificação das empresas e, uma foi organizada para compreender o uso das mídias sociais, estruturada em escala Likert de 5 pontos, onde 1 significa "nenhum uso" e 5 "máximo de uso". Outras 21 assertivas foram elaboradas, de forma que os respondentes escolhiam uma posição (pontuação) de 0 a 100, em uma barra contínua, onde 0 significava "reduziu $10 \%$ ou mais", 50 indicava "não reduziu nem aumentou" e 100 apontava que "aumentou $10 \%$ ou mais". A Figura 2 refere-se a uma das assertivas do questionário e mostra como as mesmas foram organizadas.

\section{Figura 2 - Esboço da formulação das assertivas}

* 8 O monitoramento das mídias sociais da nossa empresa:

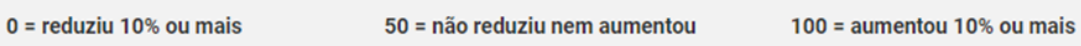

Fonte: os autores (2018).

O instrumento resultante foi então submetido a um pré-teste com 3 profissionais e, posteriormente, validado por especialista da área. Após a validação, o questionário foi enviado por e-mail para a população de amostra.

Entende-se que essas variáveis estabelecem indicadores importantes, pois criam a possibilidade de comparar e medir o desempenho das empresas. Portanto, o questionário foi organizado em quatro partes: a primeira serviu para caracterizar o respondente; a segunda buscou identificar a utilização das MS pelas empresas; a terceira procurou identificar as CD presentes nas empresas; e a quarta buscou medir o DM de cada empresa.

Procedeu-se o tratamento dos dados por meio dos testes Kolmogorov-Smirnov e Shapiro-Wilk para verificar a normalidade. Posteriormente a análise fatorial exploratória (AFE) para avaliar a dimensionalidade das escalas, usando o software SPSS $®$. Em seguida realizouse a modelagem de equações estruturais (SEM), utilizando o software AMOS ${ }^{\circ}$. Foram testadas como variáveis de controle tamanho da empresas e setor. Para isto foram efetuadas análises de

\section{Organizadores:}

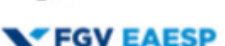

\section{CONTRO of}

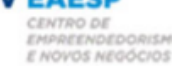

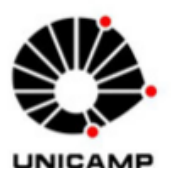
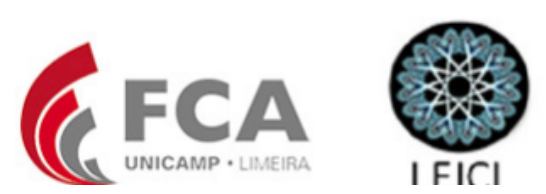
regressão e análise de variância - ANOVA, tendo como independentes as variáveis de controle, sendo que não foi localizada nenhuma influência destas sobre os construtos estudados.

\section{Resultados, análises e discussões}

Os micros, pequenos e médios negócios empresariais no Brasil constituíram no ano de 2015, uma população com mais de 6,8 milhões de estabelecimentos (SEBRAE, 2017). Nesse conjunto empresarial, a imersão desses atores nas MS acaba sendo um diferencial, especialmente para os que desejam melhorar seu desempenho mercadológico. Para facilitar a análise dos dados, referentes às MPMEs participantes desta pesquisa, procedeu-se primeiramente, uma análise descritiva dos dados coletados, e posteriormente, por meio da modelagem de equações estruturais, foram testadas as hipóteses formuladas.

\subsection{Análise Descritiva dos Dados}

Entre a amostra pesquisada (serviço, comércio e indústrias), o setor de serviços possui o maior quantitativo de empresas, seguido pelo comércio e pela indústria. Referente à atuação, destacam-se as micro empresas que, nos três setores somam $65,03 \%$, enquanto as pequenas atingem $29,37 \%$ e as médias $5,60 \%$. Por setor, a indústria, com 52,67\% apresentou o maior número de funcionários, seguida pelo serviço, com $31,62 \%$, e pelo comércio com $15,71 \%$. Ficou evidente que as empresas utilizam, de alguma forma, mídias sociais. Com base nas respostas, a Tabela 1, retrata as médias de utilização de cada mídia, por setor, e o geral.

Tabela 1 - Média da utilização das Mídias Sociais, por setor de atuação e média geral.

\begin{tabular}{|c|c|c|c|c|c|c|c|c|c|c|c|c|c|}
\hline 苛 & 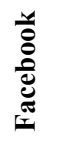 & 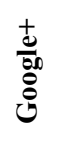 & 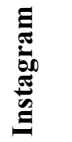 & 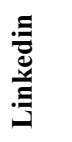 & 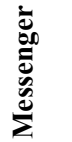 & 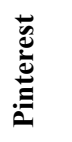 & $\frac{\check{L}}{\mathscr{a}}$ & 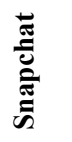 & 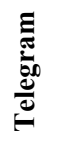 & 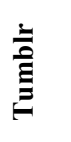 & 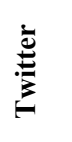 & 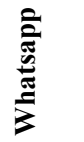 & 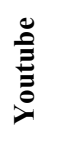 \\
\hline Comércio & 4,03 & 2,12 & 2,88 & 1,50 & 2,41 & 1,12 & 2,15 & 1,24 & 1,12 & 1,09 & 1,50 & 4,38 & 1,79 \\
\hline Indústria & 3,16 & 1,89 & 1,95 & 2,42 & 1,89 & 1,00 & 3,00 & 1,00 & 1,00 & 1,00 & 1,21 & 3,95 & 1,79 \\
\hline Serviço & 3,77 & 2,21 & 2,62 & 1,97 & 2,28 & 1,31 & 2,57 & 1,20 & 1,12 & 1,01 & 1,47 & 4,38 & 2,00 \\
\hline Geral & 3,65 & 2,07 & 2,48 & 1,96 & 2,19 & 1,14 & 2,57 & 1,15 & 1,08 & 1,03 & 1,39 & 4,24 & 1,86 \\
\hline
\end{tabular}

Fonte: Dados da pesquisa (2018).

Os respondentes selecionaram entre 1 a 5 , a resposta que melhor representava sua realidade, onde 1 significou "nenhum uso daquela mídia social" e 5 "máximo de uso daquela mídia social". A média geral para a utilização de cada mídia demonstra que a maior utilização é do Whatsapp, seguido pelo Facebook. E apresentam menor utilização o Tumblr, Telegram, Pinterest e Snapchat. Além de analisar a utilização das MS, também foi possível identificar alguns aspectos referentes a cada um dos três constructos da pesquisa.

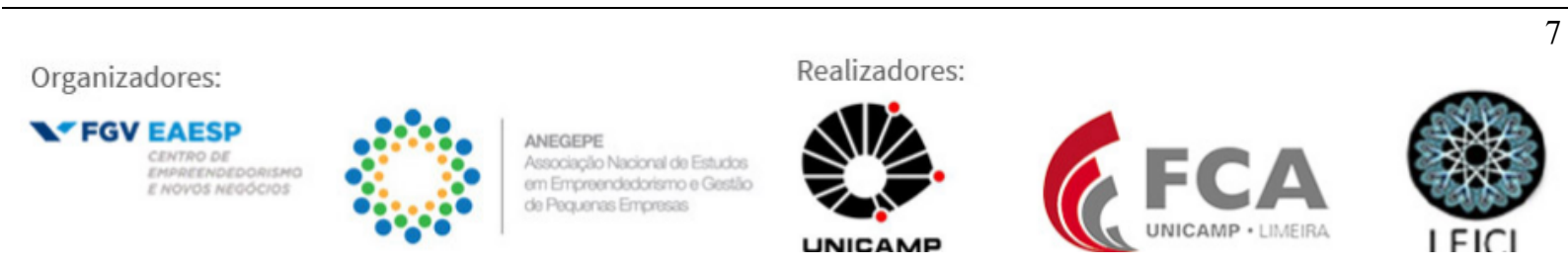


O constructo "Mídias Sociais" [MS] foi identificado com oito assertivas, cujos resultados indicaram as médias das respostas acima dos 50 pontos, com indicativos de que as MPMEs de SC, aumentaram o uso das mídias sociais em 2017, em relação ao ano anterior. O maior crescimento refere-se à divulgação dos produtos ou serviços nas MS, que obteve a maior média $(74,68)$. O menor refere-se ao trabalho para garantir que os recursos da MS não fossem facilmente identificados ou imitados pelos concorrentes que ficou com média de 53,14.

O constructo "Capacidades Dinâmicas" [CD] abarcou oito assertivas, que também atingiu média acima os 50 pontos. Isso indica que as empresas, de alguma forma, buscaram desenvolver ou reconfigurar competências internas e externas objetivando adaptar-se às mudanças (TEECE; PISANO; SHUEM, 1997) e alcançar vantagem competitiva (LIN, WU, 2014). A agilidade de resposta às mudanças do ambiente competitivo, usando as mídias sociais, foi a variável com maior média $(70,66)$. A menor média $(51,13)$ refere-se ao uso das mídias sociais, na capacidade de infraestrutura de TI.

O terceiro constructo "Desempenho Mercadológico" [DM], reflete cinco assertivas e na média, indica que as MPMEs conseguiram alcançar resultados positivos. Entender o DM é importante, pois auxilia as MPMEs a identificar ameaças e oportunidades, a manter atenção às mudanças no mercado (CHENHALL; MOERS, 2015) e ainda a visualizar as oportunidades de inovação do negócio. Em relação ao ano de 2016, o maior crescimento foi em relação ao nível de satisfação dos clientes, com média de 70,73 e o menor crescimento foi do nível de retenção dos clientes com média de 63,10. A Tabela 2 apresenta os valores descritivos de cada indicador.

Tabela 2 - Variáveis e descrição dos dados da pesquisa

\begin{tabular}{|c|c|c|c|c|c|c|}
\hline Constructo & Variáveis & M & DP & Variação & Assimetria & Curtose \\
\hline \multirow{8}{*}{$\begin{array}{c}\text { Mídias } \\
\text { Sociais (MS) }\end{array}$} & Atuação da empresa nas MS & 72,31 & 26,31 & 692,39 &,- 871 & ,268 \\
\hline & Utilização de informações das MS para conhecer cliente & 66,65 & 24,04 & 577,93 &,- 188 &,- 515 \\
\hline & Monitoramento das MS & 69,83 & 26,08 & 679,92 &,- 709 &, 195 \\
\hline & Planejamento das estratégias para MS da empresa & 69,06 & 25,54 & 652,23 &,- 605 & 103 \\
\hline & Interação com o consumidor por meio das MS & 74,07 & 25,18 & 634,02 &,- 838 & ,369 \\
\hline & Ações da empresa a partir dos resultados das MS & 64,91 & 24,91 & 620,51 &,- 358 &,- 138 \\
\hline & Investimento em MS pela empresa & 62,49 & 27,17 & 738,24 &,- 352 &,- 184 \\
\hline & Divulgação dos prod./serv. nas MS & 74,68 & 25,66 & 658,60 &,- 930 &, 407 \\
\hline \multirow{8}{*}{$\begin{array}{c}\text { Capacidades } \\
\text { Dinâmicas } \\
\text { (CD) }\end{array}$} & $\begin{array}{l}\text { Trabalho para que os recursos de MS não fossem } \\
\text { identificados/imitados }\end{array}$ & 53,14 & 25,26 & 638,16 &,- 012 &, 242 \\
\hline & $\begin{array}{l}\text { Compartilhamento das informações das MS entre setores } \\
\text { da empresa para visão integrada }\end{array}$ & 59,19 & 28,49 & 811,59 &,- 281 &,- 341 \\
\hline & $\begin{array}{l}\text { Com uso MS reorganização da equipe de negócios ou } \\
\text { competências internas }\end{array}$ & 57,52 & 24,61 & 605,50 &,- 177 & ,423 \\
\hline & Com uso MS, a capacitação da infraestrutura de TI & 51,13 & 23,25 & 540,34 &,- 217 & ,966 \\
\hline & Com uso MS pressão do concorrente & 58,57 & 22,71 & 515,87 &,- 120 &, 731 \\
\hline & Com uso MS, esforço quanto ao gerenciamento de MKT & 65,89 & 27,10 & 734,58 &,- 657 & ,204 \\
\hline & Com uso MS esforço quanto a gestão da inovação & 67,10 & 24,99 & 624,70 &,- 472 &, 152 \\
\hline & $\begin{array}{l}\text { Com uso MS agilidade de resposta às mudanças } \\
\text { do ambiente competitivo }\end{array}$ & 70,66 & 24,66 & 608,25 &,- 646 & ,201 \\
\hline \multirow{5}{*}{$\begin{array}{c}\text { Desempenho } \\
\text { Mercadológi } \\
\text { co (DM) }\end{array}$} & Nível satisfação clientes & 70,73 & 21,45 & 460,07 &,- 612 & 679 \\
\hline & Nível retenção clientes & 63,10 & 24,09 & 580,54 &,- 366 & ,272 \\
\hline & Número de novos clientes & 67,87 & 25,41 & 645,46 &,- 695 & ,466 \\
\hline & Faturamento da empresa & 63,64 & 24,33 & 592,02 &,- 281 & 334 \\
\hline & Volume de vendas da empresa & 65,12 & 24,32 & 591,53 &,- 442 &, 415 \\
\hline
\end{tabular}

Fonte: Dados da pesquisa (2018). 


\subsection{Análise Exploratória dos Dados}

Como os dados aproximam-se da normalidade, com valores de curtose e assimetria relativamente baixos, antes de proceder ao teste de hipóteses, por meio da Modelagem de Equações Estruturais (SEM), optou-se por realizar a análise fatorial exploratória (AFE), objetivando avaliar a dimensionalidade das escalas. A AFE é utilizada em estudos com constructos reflexivos, reunindo as variáveis no número de fatores ideal para melhor representar os dados (HAIR et al., 2009). Foi utilizado o método de componentes principais e o critério eigenvalue igual a 1 (um) que oportunizaram extrair os fatores. Para adequação correta do método, as variáveis "Planejamento das estratégias para MS da empresa", "Ações da empresa a partir dos resultados das MS", "Investimento em MS pela empresa", "Com uso MS pressão do concorrente" e "Com uso MS agilidade de resposta às mudanças do ambiente competitivo", precisaram ser excluídas.

O grau de explicação do Kaiser-Meyer-Olkin (KMO) ficou em 0,894, e o valor do Qui Quadrado do teste de Bartlett foi de 1813,08, significante a 0,000, o que indica adequabilidade dos dados (Hair et al., 2009). A variância explicada foi de $67,05 \%$ atendendo aos valores apontados na literatura (HAIR et al., 2009). Outros valores estão apresentados na Tabela 3, onde foram suprimidos as cargas com valor abaixo de 0,455 , dentro do estipulado (HAIR et al., 2009) para seguir a análise.

Tabela 3 - Constructos, variáveis, fatores, cargas fatoriais e comunalidades

\begin{tabular}{|c|c|c|c|c|c|}
\hline \multirow{2}{*}{ Constructo } & \multirow{2}{*}{ Variáveis } & \multicolumn{3}{|c|}{ Componentes } & \multirow{2}{*}{$\begin{array}{c}\text { Comuna } \\
\text { lidade }\end{array}$} \\
\hline & & 1 & 2 & 3 & \\
\hline \multirow{6}{*}{ MS } & Atuação da empresa nas MS & & &,- 775 & ,745 \\
\hline & Utilização de informações das MS para conhecer cliente & & &,- 831 & 617 \\
\hline & Monitoramento das MS & & &,- 706 & ,686 \\
\hline & Interação com o consumidor por meio das MS & & &,- 540 & 669 \\
\hline & Ações da empresas a partir dos resultados das MS & & &,- 550 & ,750 \\
\hline & Divulgação dos prod./serv. nas MS & & &,- 529 & 697 \\
\hline \multirow{7}{*}{$\mathrm{CD}$} & Trabalho para que os recursos de MS não fossem identificados/imitados & ,696 & & &, 595 \\
\hline & $\begin{array}{l}\text { Compartilhamento das informações das MS entre setores da empresa para visão } \\
\text { integrada }\end{array}$ & ,764 & & & ,586 \\
\hline & Com uso MS reorganização da equipe de negócios ou competências internas & ,789 & & & ,721 \\
\hline & Com uso MS, a capacitação da infraestrutura de TI &, 850 & & & 648 \\
\hline & Com uso MS, a pressão do concorrente & 629 & & & 457 \\
\hline & Com uso MS, esforço quanto ao gerenciamento de MKT & 668 & & & ,702 \\
\hline & Com uso MS esforço quanto a gestão da inovação & ,666 & & & 625 \\
\hline \multirow{5}{*}{$\mathrm{DM}$} & Nível satisfação clientes & & ,706 & & ,656 \\
\hline & Nível retenção clientes & & ,758 & & 651 \\
\hline & Número de novos clientes & & 807 & & ,749 \\
\hline & Faturamento da empresa & & 912 & &, 797 \\
\hline & Volume de vendas da empresa & & 843 & &, 718 \\
\hline
\end{tabular}

Método de extração: Análise do Componente principal.

Método de rotação: Oblimin com normalização de Kaiser.

Fonte: Dados da pesquisa (2018). 
Em seguida foi realizado o teste de confiabilidade por meio do Alpha de Cronbach para os três constructos. O Alfa de Cronbach para os constructos MS, CD e DM foi, respectivamente de, 0,912, 0,890, e 0,893. Assim, de acordo com o recomendado pela literatura (HAIR et al., 2009), as medidas de consistência interna e de confiabilidade do modelo estão adequadas para seguir com a realização da Modelagem de Equações Estruturais.

\subsection{Modelagem de Equações Estruturais}

Após alguns testes, chegou-se ao modelo mais ajustado, composto por sete indicadores para o constructo CD, sete para MS e cinco para DM, conforme o indicado na Figura 3.

Figura 3 - Variáveis e descrição dos dados da pesquisa

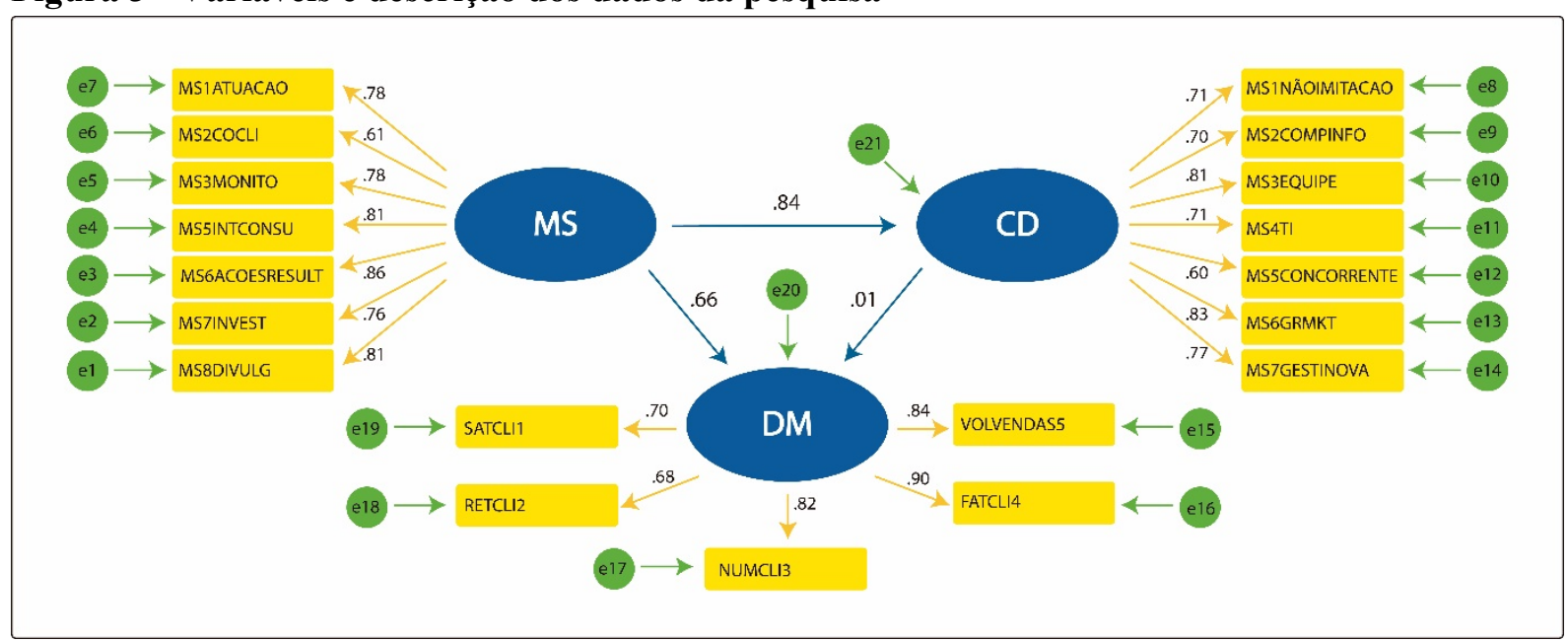

Fonte: Dados da pesquisa (2018).

Os indicadores de ajuste geral do modelo ficaram em 2,537 para o valor de CMIN/DF, 0,877 para o CFI, e 0,104 para o RMSEA, valores que demonstram que o ajuste do modelo não é o ideal, mas é razoável, conforme o preconizado por Hair et al. (2009).

A última etapa consistiu em analisar as hipóteses de pesquisa e os testes de significância. Os coeficientes calculados para as relações entre os constructos previstos nas hipóteses H1 e $\mathrm{H} 2$ foram aceitos ( $p$-value=0,000). Assim, foi confirmado que MS influenciam CD e DM. Já a hipótese $\mathrm{H} 3$ foi rejeitada identificando que DM não sofre influencia de $\mathrm{CD}$, conforme indicado na Tabela 4.

Tabela 4 - Resultados dos testes de significância dos modelos de pesquisa

\begin{tabular}{cccccccc}
\hline Hipótese & Variável Dependente & & Variável Independente & Estimativa & p-value & C.R. & Status \\
\hline H1 & MS & $--->$ & CD & .842 & 0,000 & 6.409 \\
H2 & MS & $--->$ & DM & .657 & 0,000 & 3.646 & Suportada \\
H3 & CD & $--->$ & DM & .007 & 0.691 & 0.420 & Rejeitada \\
\hline
\end{tabular}

Fonte: Dados da pesquisa (2018) 


\subsection{Discussãa dos resultados}

Os resultados deste estudo assinalam que as MS geram CD e também DM, nas MPMEs investigadas, contudo, quando as MS são utilizadas mediadas pelas $\mathrm{CD}$, não há influência no DM, ou seja, o uso das MS não gera DM de maneira indireta por meio das CD. As empresas participantes deste estudo, confirmam as considerações de Teece et al., (1997) quanto este afirma que as empresas exploram suas competências internas e externas para enfrentar o ambiente de mudança. Então, as MS geram CD, o que ratifica a $\mathrm{H} 1$ proposta "MS gera $C D$ " estatisticamente. Os resultados aproximam-se daqueles indicados por Lam et al., (2016) ao considerarem que as mídias sociais possibilitam a melhoria das rotinas dinâmicas, conquistando melhor desempenho.

Existem indicativos de que as empresas analisadas precisam internalizar o uso das mídias sociais como possibilidades de conhecer e se relacionar melhor com seus stakeholders. A imersão de empresas nas MS pode mudar sua configuração de relacionamento com seus stakeholders, pois fornecem uma plataforma para compartilhamento de experiências, acumulação de conhecimento e aprendizado organizacional (NGUYEN et al., 2015; LAM et al., 2016). As MPMEs, participantes do estudo, no ano de 2017, aumentaram timidamente o compartilhamento de experiências, a utilização das informações para conhecer os clientes e também as ações a partir das informações das MS.

No constructo MS, as pequenas empresas possuem médias de atuação superior nas mídias sociais, seguida pelas microempresas. Tal resultado aproxima-se daquele encontrado por Braojos-Gomez et al. (2015), onde descobriu em sua análise empírica, que a competência das redes sociais é mais importante para as empresas de menor tamanho. Uma explicação pode ser o fato de que por possuírem menor portfólio de recursos financeiros, nas pequenas empresas as capacidades de mídias tornam-se mais importantes que para as empresas maiores (BRAOJOS-GOMES et al., 2015).

Os resultados dos cálculos estatísticos suportaram a hipótese H2 de que "a utilização de MS gera desempenho". Tal resultado reflete e confirma a teoria de Venkatraman e Tamanujam, (1986) e Callado et. al., (2008) quanto a identificação e mensuração do uso das MS nas empresas para motivar o desempenho. Os resultados desta pesquisa aproxima-se ao que indicam os autores Lombradi et al., 2010 e também Santos et al., 2014, quando estes indicam que as empresas atuam em ambientes competitivos e de incertezas e que as decisões tomadas pelos gestores possibilitam atingir maior ou menor lucratividade, crescimento nas vendas e conquistar clientes. Este estudo evidencia de que embora as MPMEs catarinenses estejam preocupadas em melhorar o faturamento, reter clientes, e melhorar o faturamento, ainda é possível melhorar seu desempenho.

Alguns estudos corroboram com tal visão ao indicarem que para atingir DM, as empresas necessitam desenvolver $\mathrm{CD}$, ser capazes de atrair novos clientes, reter os atuais, 
aumentar a lucratividade, bem como a participação no mercado, além de oferecer maior qualidade, preços atraentes e atendimento personalizado (CHRISTENSEN, 1997; OECD, 2005; TIDD et al., 2005; TIGRE, 2006; CHESBROUGH, 2012; TEECE et al., 2014).

Os achados deste estudo ainda aproximam-se daqueles encontrados por Braojos-Gomes et al., 2015 ao sugerir que a capacidade da infraestrutura de TI, a pressão do concorrente social, o gerenciamento de marketing e a gestão da inovação são mecanismos fundamentais através dos quais as pequenas empresas aprendem a desenvolver uma competência em redes sociais.

Os resultados encontrados neste estudo, onde a hipótese $\mathrm{H} 3$ "CD estão positivamente relacionadas com desempenho, isto é, CD geram DM", não foi suportada, também aproximase das evidências empíricas de Braojos-Gomez et al. (2015). Isso porque os mecanismos fundamentais através dos quais as MPMEs aprendem a desenvolver competências em redes sociais, precisam se desenvolver nessas empresas. Os resultados apontaram que a capacidade da infra-estrutura de TI por exemplo, não aumentou nem reduziu em 2017, a pressão do concorrente social aumentou pouco e o gerenciamento de marketing e a gestão da inovação aumentaram levemente.

Os resultados também se aproximam da afirmação de Teece et al. (2014) de que a criação de riqueza em regimes de alta mudança tecnológica dependem em grande medida da capacidade tecnológica, organizacional e de processos gerenciais da empresa. Ainda, as empresas estudadas sentem dificuldades em transformar os dados das MS em conhecimentos pessoais valiosos e explorar esses insights para criar valor agregado para os clientes (WIENEKE; LEHRER, 2016). Assim sendo, entende-se que a validade estatística da hipótese não se confirma, pois as empresas estudas necessitam fomentar suas CD.

A teoria vislumbra que as $\mathrm{CD}$ possibilitam desenvolver diferenciais de mercado, frente à concorrência (EISENHARDT; MARTIN, 2014). Os resultados deste estudo, indicam que as MPMEs do estado de SC buscam tais diferenciais, mas ainda há espaço para melhorar. Isso se torna visível, ao analisar a média de pontuação da variável "trabalho para que os recurso de MS não sejam identificados/imitados", bem como a variável "com uso de MS a pressão do concorrente". Portando, as empresas parecem não utilizar ou desconhecem as CD como recurso para melhorar seu DM, (EISENHARDT; MARTIN, 2014). Ainda, a relação das CD com a vantagem competitiva, está fundamentada na possibilidade de a empresa criar, configurar, integrar e combinar seus recursos (EISENHARDT; MARTIN, 2014) e isso tem a ver com a maturidade da organização, pois isso impacta no DM. (BARRA; LADEIRA, 2017; BARNEY; HERTERLY, 2007).

Por fim, Barra e Ladeira, (2017) e Barney e Herterly, (2007) afirmam que estratégias diferenciadas (capacidades e recursos raros, inimitáveis, de valor, e organizados) podem gerar valor ao cliente, e obter desempenho superior. Tal afirmativa colabora para o entendimento de que as MPMEs do Estado de SC, Brasil, necessitam, ainda, absorver e aprender com suas CD.

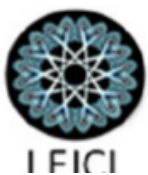




\section{Conclusão}

O objetivo principal do estudo foi analisar o impacto do uso das mídias sociais nas capacidades dinâmicas e no desempenho mercadológico das micro, pequenas e médias empresas atuantes no estado de Santa Catarina, Brasil. Visando testar a relação entre os constructos, aplicou-se uma survey para gestores das empresas do Estado de Santa Catarina, Brasil, 143 respostas. A estrutura do estudo integra e sintetiza conceitos e resultados da pesquisa de campo e os resultados mostram que MS propicia $\mathrm{CD}$, que MS gera $\mathrm{DM}$, mas que CD não geram DM.

As capacidades das MS podem ser mais importantes às pequenas empresas, que para as grandes. Contudo, devido às possibilidades de recursos financeiros, o modelo demonstra que as MPMEs necessitam desenvolver maior absorção das CD, para que a eficácia de DM, nas MS se concretize com maior robustez. Os achados deste estudo confirmam que as empresas têm dificuldades quanto a capacidade de infraestrutura de TI, quanto a pressão de concorrente social e quanto as capacidades de marketing e gerenciamento de inovação. Esses são mecanismos fundamentais através dos quais as empresas aprendem a desenvolver uma competência em redes sociais. (BRAOJOS-GOMEZ et al., 2015).

O estudo também recomenda que a competência das MS é mais importante para as empresas mais pequenas. Entende-se que todos os mecanismos citados (a capacidade de infraestrutura de TI, a pressão social dos concorrentes, o gerenciamento de marketing e a gestão da inovação) são antecedentes fundamentais através dos quais as pequenas empresas aprendem a desenvolver uma competência em redes sociais, apesar deste estudo não ter testado a ordem de importância desses mecanismos (BRAOJOS-GOMEZ et al., 2015).

Este estudo demonstrou que as MPMEs necessitam melhorar suas CD. Isto é, buscar absorver as informações do mundo digital e transformar os dados das MS em conhecimentos pessoais e valiosos, a fim de, como afirmam Wieneke e Lehrer (2016) explorar os insights para criar valor agregado para os clientes. O estudo ainda contribuiu ao indicar, na mesma percepção de Teece et al;. (2014) que, a criação de riquezas em regimes de alta mudança tecnológica depende, em grande medida, da capacidade tecnológica, organizacional e de processos gerenciais da empresa.

Conclui-se que as MPMEs do estado de Santa Catarina, estão em um processo de amadurecimento, da utilização de mídias sociais para gerar capacidades dinâmicas e desempenho mercadológico. Apresentam avanços, contudo ainda podem aprimorar-se mais, desenvolvendo capacidades dinâmicas que possibilitem maior desempenho. É justamente na geração de desempenho, a partir das capacidades dinâmicas que parecem residir os maiores desafios. Relembra-se que são os gestores, os responsáveis, a partir de suas decisões, pelo desenvolvimento interno e conquista de mercado.

\section{Limitações de pesquisa e sugestões de pesquisas futuras}

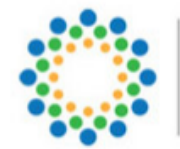

ANEGEPE
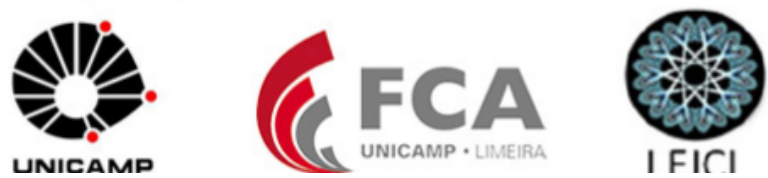
Embora o modelo proposto explique questões referentes às MPMEs do estado de SC, pode haver fatores métricos nas MS dessas empresas ou percepções dos seus stakeholders, que influenciam nas CD e no DM das MS. Ainda é preciso considerar que este estudo concentra-se em percepções dos gestores das empresas, havendo incentivo para que pesquisadores busquem ampliar a coleta de dados com funcionários e clientes, com possibilidade de utilizar dados secundários, sobre as mídias sociais das empresas pesquisadas. Outra questão refere-se ao fato do estudo ter abarcado micro, pequenas e médias empresas. Talvez com uma só categoria, os resultados sejam distintos. Finalmente, considera-se interessante realizar pesquisas adicionais para entender como as capacidades das mídias sociais influenciam o desempenho de grandes empresas em suas inovações.

\section{Referências Bibliográficas}

AHANI Ali, RAHIM, Nor Zairah Ab.; Nilashi Mehrbakhsh. Forecasting social CRM adoption in SMEs: A combined SEM-neural network method. Computers in Human Behavior. v. 75, p. 560-578 19 p., 2017.

BARNEY, J. Firm resources and Sustained Competitive Advantage. Journal of Management, v. 17, n. 1, p. 99-120, 1991.

BARNEY, J.B; HESTERLY, W.S. Administração Estratégica e Vantagem Competitiva: casos brasileiros. São Paulo: Pearson, 2007.

BARRA, G. M. J.; LADEIRA, M. B. Modelo de maturidade para processos de certificação no sistema agroindustrial do café. REGE-Revista de Gestão, v. 24, p. 134-148, 2017.

BRAGANZA, Ashley; Brooks, Laurence; Nepelski, Daniel; Ali, Maged; Moro, Russ. Resource management in big data initiatives: Processes and dynamic capabilities. Journal of Business Research, v. 70, p. 328-337, 2017.

BRAOJOS-GOMEZ, Jessica; BENITEZ-AMADO, Jose; LLORENS-MONTES, F. Javier. How do small firms learn to develop a social media competence? International Journal of Information Management, 35 443-458, 2015.

CAllado, A. A. C.; CALlADO, A. L. C.; ANDRADE, L. P. Padrões de utilização de indicadores de desempenho não-financeiros: um estudo exploratório nas empresas de Serra Talhada/PE. ABCustos, v. 3, n. 2, p. 1-26, mai./ago. 2008.

CASTELLS, Manuel. A galáxia da Internet. Rio de Janeiro: Jorge Zahar, 2003;

CHENHALL, R. H.; MOERS, F. The role of innovation in the evolution of management accounting and its integration into management control. Accounting, Organizations and Society, v. 47, p. 1-13, 2015.

CHESBROUGH, H. Inovação aberta: como criar e lucrar com a tecnologia. Porto Alegre: Bookman, 2012.

CHRISTENSEN, C. M. The innovator's Dilemma: when new technologies cause great firms to fail. Boston, Massachusetts: Harvard Business School Press, 1997.

EISENHARDT, K. M.; MARTIN, J. A. Dynamic capabilities: what are they? Strategic Management Journal, v. 21, n. 10-11, p. 1105-1121, oct. 2000.
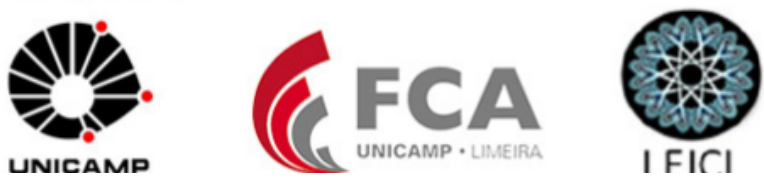
EISENHARDT; MARTIN, 2014. O que são as capacidades dinâmicas? In: LACERDA, Daniel Pacheco; TEIXEIRA, Rafael; ANTUNES, Junico; CORCINI NETO, Secundino Luis. Estratégia Baseada em Recursos. Porto Alegre: Bookman, 2014.

EREVELLES, Sunil; FUKAWA, Nobuyuki; SWAYNE, Linda. Big Data consumer analytics and the transformation of marketing, Journal of Business Research. Journal of Business Research, vol. 69, issue 2, 897-904, 2016.

GUPTA, Manjul; GEORGE, Joey. Toward the development of a big data analytics capability. Information \& Management, v. 53 8, p. 1049-1064, 2016.

HAIR JR., J.F.; WILLIAM, B.; BABIN, B.; ANDERSON, R.E. Análise multivariada de dados. 6.ed. Porto Alegre: Bookman, 2009.

LAM, Hugo K.S., YEUNG, Andy C.L.; CHENG, T.C. Edwin. The impact of firms' social media initiatives on operational efficiency and innovativeness. Journal of Operations Management. 47-48:28-43, 2016.

LIN, Y.; WU, L. Y. Exploring the role of dynamic capabilities in firm performance under the resource-based view framework. Journal of Business Research, v. 67, n. 3, p. 407-413, 2014. LI, Liang; SU, Fang; ZHANG, Wei; MAO, Ji-Ye. Digital transformation by SME entrepreneurs: A capability perspective. Info Systems J. 0:1-29, 2017.

LIU, Yong; Deng, Shengli Hu, Feng; Chen, Xiaoyu. The impacts of unique service resources and habit on e-service loyalty in a highly competitive market. Journal of Systems and Information Technology, v. 17, 4, p. 336-350, 2015.

MARTYNOW, A.; SHAFTI, N. Long-term performance of firms: a review and research agenda. Journal of Strategic anda Management. v. 9, n. 4, p. 429-448, 2016.

NGUYEN, Bang; YU, Xiaoyu; MELEWAR, T.C.; CHEN, Junsong. Brand innovation and social media: knowledge acquired from social media, market orientation, and the moderating role of social media strategic capability. Industrial Marketing Management. 51 . pp. 11-25, 2015.

OECD. Modernising Government: the way forward. Paris, OECD Publishing: 2005.

PETERAF, M. A. The cornerstones of competitive advantage: a resource-based view. Strategic Management Journal. v. 14, n.3, p. 179-191, mar. 1993.

PINHO, José Carlos; PRANGE, Christiane. The effect of social networks and dynamic internationalization capabilities on international performance. Journal of World Business. v. 51 3, p. 391-403, 2016.

POGREBNYAKOV, Nicolai. A cost-based explanation of gradual, regional internationalization of multinationals on social networking sites. Management International Review, v. 57 1, 2017.

PORTER, M.E. Competitive Strategy: Techniques for Analyzing Industries and Competitors. New York: Free Press, 1980.

SANTOS, N. M.; BRONZO, M.; OLIVEIRA, M. P. V.; RESENDE, P. T. V. Cultura organizacional, estrutura organizacional e gestão de pessoas como bases para uma gestão orientada por processos e seus impactos no desempenho organizacional. Brazilian Business Review. v. 11, n. 3, p. 106-129, maio-jun. 2014.

SEBRAE - Serviço Brasileiro de Apoio às Micro e Pequenas Empresas. Anuário do trabalho na micro e pequena empresa 2015. 8 ed. Brasília: DIEESE, 2017.
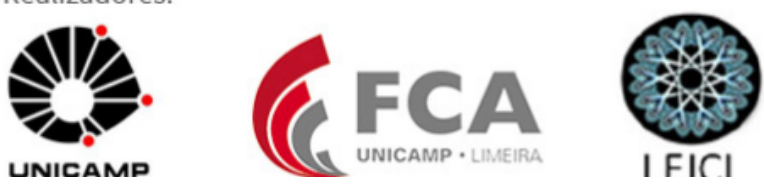
TEECE, D. J.; PISANO, G.; SHUEN, A. Dynamic capabilities and strategic managment. Strategic Managment Journal. v. 18, n. 7, p. 509-533, Aug. 1997.

TEECE, David; PISANO, Gary; SHUEN, Amy. As Capacidades dinâmicas e gestão estratégica. In: LACERDA, Daniel Pacheco; TEIXEIRA, Rafael; ANTUNES, Junico; CORCINI NETO, SecundinoLuis. Estratégia Baseada em Recursos. Porto Alegre: Bookman, 2014.

TELLES, André. A revolução das mídias sociais: cases, conceitos, dicas e ferramentas. São Paulo:M. Books, 2011.

TIDD, J.; BESSANT, J.; PAVITT, K. Managing innovation: integrating technological, market and organizational change. ed.3. Chichester: John Wiley \& Sons, 2005.

VENKATRAMAN, N.; TAMANUJAM, V. Measurement of business performance in strategy research: a comparison of approaches. Academy of Management Review. v. 1, n. 4, p. 801814, 1986.

WAMBA, Samuel Fosso; GUNASEKARAN, Angappa; AKTER, Shahria; REN, Steven Ji-fan; DUBEY, Rameshwar; CHILDE, Stephen J.. Big data analytics and firm performance: Effects of dynamic capabilities. Journal of Business Research. v. 70 p. 356-365, 2017.

WANG, C. L.; AHAMED, P. K. Diynamic capabilities: a review and research agenda. International Journal of Management Reviews. v. 9, n. 1, p. 31-51, 2007.

WANG, Zhan; KIM, Hyun Gon. Can Social Media Marketing Improve Customer Relationship Capabilities and Firm Performance? Dynamic Capability Perspective. Journal of Interactive Marketing. v. 39 p. 15-26, 2017.

WIENEKE, Alexander; LEHRER, Lehrer. Generating and exploiting customer insights from social media data. Electron Markets. 26:245-268, 2016.

ZAVATTARO, SS; DASPIT, JJ. Branding and Isomorphism: The Case of Higher Education. Journal of Vacation Marketing 22 (4), 349-364, 2016.

ZOLLO, M.; WINTER, S. G. Deliberate Learning and the Evolution of Dynamic Capabilities. Organization Science. v. 13, n. 3, p. 339-351, mai./jun. 2002. 